\title{
Contactless Readout of Passive LC Sensors with Compensation Circuit for Distance-Independent Measurements ${ }^{\dagger}$
}

\author{
Marco Baù *, Marco Demori, Marco Ferrari and Vittorio Ferrari \\ Department of Information Engineering, University of Brescia, 25123 Brescia, Italy; \\ marco.demori@unibs.it (M.D.); marco.ferrari@unibs.it (M.F.); vittorio.ferrari@unibs.it (V.F.) \\ * Correspondence: marco.bau@unibs.it; Tel.: +39-030-371-5896 \\ † Presented at the Eurosensors 2018 Conference, Graz, Austria, 9-12 September 2018.
}

Published: 3 December 2018

\begin{abstract}
Contactless readout of passive LC sensors composed of a capacitance sensor connected to a coil can be performed through an electromagnetically coupled readout coil set at distance $d$. Resonant frequency $f_{s}$ and Q-factor $Q s$ of the LC sensor can be extracted from the measurement of the impedance at the readout coil by using a technique theoretically independent of $d$. This work investigates the effects on the measurement accuracy due to the unavoidable parasitic capacitance $C_{P}$ in parallel to the readout coil, which makes the measured values of $f_{\mathrm{s}}$ and $Q_{s}$ dependent on $d$. Numerical analysis and experimental tests confirm such dependence. To overcome this limitation, a novel electronic circuit topology for the compensation of $C_{P}$ is proposed. The experimental results on assembled prototypes show that for a LC sensor with $f_{s} \approx 5.48 \mathrm{MHz}$ a variation of less than 200 ppm across an interrogation distance between 2 and $18 \mathrm{~mm}$ is achieved with the proposed compensation circuit.
\end{abstract}

Keywords: distance-independent contactless readout; LC resonant sensors; telemetric sensors

\section{Introduction}

Passive LC sensors can be obtained by connecting a capacitive sensor to a coil, thus forming a resonant LC circuit where the measurement information is related to the resonant frequency and the quality factor of the LC circuit. By magnetically coupling the coil connected to the sensor to an external interrogation coil, contactless readout of the LC-sensor can be achieved [1]. This configuration has recently gained particular interest in several fields such as industrial, chemical or biomedical applications. The lack of wired connection to the sensor is attractive in applications where cabled solutions are difficult or unpractical, such as measurement in sealed environments or inside animal/human bodies [1,2]. Additionally, these applications can take advantage on the absence of on-board power supply, allowing in principle for unattended unlimited operation. In-field measurement conditions are typically characterized by uncontrolled distance between the readout and the sensor coils, thus requiring interrogation techniques that desirably are independent of the distance between the coils. Approaches theoretically independent of the distance have been proposed both in the time-domain [3,4] and in the frequency-domain [2]. In the latter case, measuring the real part of the impedance at the readout coil allows to obtain the resonant frequency and the quality factor of the LC sensor $[5,6]$. The present work investigates on the limitations of such technique when considering unavoidable parasitic capacitances in parallel to the readout coil due to the connected electronics and cables. By means of numerical simulations and experimental tests, it 
has been demonstrated that the parasitic capacitances introduce dependence on the distance. As a solution, a circuit is proposed to compensate for this unwanted effect.

\section{Operating Principle}

Figure 1a shows the schematic diagram of the proposed contactless readout system for capacitive sensors. It is composed of the interrogation unit made up by an impedance analyzer and the readout coil represented by inductance $L_{1}$ and series resistance $R_{1}$. The capacitance $C_{P}$ models the parasitic elements coming from the windings of the coil, cables, and any electronic circuits connected in parallel to the readout coil. The readout coil is magnetically coupled to the secondary coil represented by inductance $L_{2}$ and series resistance $R_{2}$. The magnetic coupling is described through the coupling factor $k= \pm M /\left(L_{1} L_{2}\right)^{1 / 2}$, where the mutual inductance $M$ is a parameter dependent on the interrogation distance $d$ between the two coils, their geometries and orientation. The secondary coil and the connected capacitive sensor $C_{S}$ form a resonant LC circuit with resonant frequency $f_{s}$ and quality factor $Q_{s}$ given by:

$$
f_{s}=\frac{1}{\sqrt{L_{2} C_{S}}}, Q_{s}=\frac{1}{R_{2}} \sqrt{\frac{L_{2}}{C_{S}}}
$$



(a)

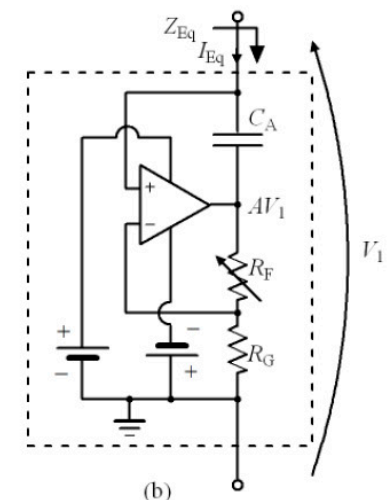

(b)

Figure 1. (a) Schematic diagram of the contactless readout system. (b) Capacitance compensation circuit.

In particular, when $C_{P}=0$ and without the compensation circuit, the sensor parameters $f_{S}$ and $Q_{S}$ can in principle be measured by considering the real part $\operatorname{Re}\left\{Z_{1}\right\}$ of the equivalent impedance $Z_{1}=V_{1} / I_{1}$ through the readout coil, which results:

$$
\operatorname{Re}\left\{Z_{1}\right\}=R_{1}+2 \pi f_{S} L_{1} k^{2} Q_{S} \frac{\left(f / f_{S}\right)^{2}}{1+Q_{S}^{2}\left(\frac{f}{f_{S}}-\frac{f_{S}}{f}\right)^{2}}
$$

From (2) it can be seen that $k$ acts only as a scaling factor and both $f_{s}$ and $Q_{s}$ can be derived considering the frequency $f_{m}$ of the maximum of $\operatorname{Re}\left\{Z_{1}\right\}$ and the Full Width at Half Maximum (FWHM) bandwidth $\Delta f_{m}$ trough the following relations [2]:

$$
f_{m}=\frac{2 Q_{S}}{\sqrt{4 Q_{S}^{2}-2}} f_{S}, Q_{S} \approx \frac{f_{S}}{\Delta f_{m}}
$$

From (3) it can be observed that for sufficiently large values of $Q_{s}$, it results $f_{m} \approx f_{s}$, obtaining a relative deviation $\left(f_{m}-f_{s}\right) / f_{s}<100 \mathrm{ppm}$ for $Q_{s}>50$. When $C_{P} \neq 0$ again, and without considering the compensation circuit, the real part of the impedance $Z_{1 P}=V_{1} / I_{1 P}=Z_{1} / / Z_{C P}$ has the following more complex expression:

$$
\operatorname{Re}\left\{Z_{1 P}\right\}=\frac{\operatorname{Re}\left\{Z_{1}\right\}}{\left(2 \pi f C_{P} \operatorname{Re}\left\{Z_{1}\right\}\right)^{2}+\left(1-2 \pi f C_{P} \operatorname{Im}\left\{Z_{1}\right\}\right)^{2}}
$$


From (3) and (4) it can be seen that now $k$ does not act only as a scaling factor and without knowing its value in advance, it is not possible to extract $f_{s}$ and $Q s$.

An analysis of the effects of $k$ and $C_{P}$ on $\operatorname{Re}\left\{Z_{1} p\right\}$ has been performed numerically. Figure 2a shows $\operatorname{Re}\left\{Z_{1 P}\right\}$ against $f / f_{s}$, i.e., the frequency normalized to $f_{s}$, for three different values of $k$ when $C_{P} / C_{S}=0.5$. The two vertical dotted lines are for $f / f_{S}=1$, i.e., corresponding to the resonant frequency of the LC sensor, and for $f / f_{S}=f_{P} / f_{S}$, i.e., corresponding to the secondary resonant frequency $f_{P}=1 /\left(2 \pi \sqrt{ }\left(L_{1} C_{P}\right)\right)$ due to $L_{1}$ resonating with $C_{P}$. When $C_{P} \neq 0$, two peaks are present in $\operatorname{Re}\left\{Z_{1} P\right\}$. Denoting with $f_{m P} / f_{s}$ the position of the maxima close to $f / f_{S}=1$ and with $f_{e P} / f_{s}$ those close to $f_{P} / f_{S}$, it can be observed that for decreasing values of $k, f_{m p} / f_{s}$ moves towards 1 and the associated peak value decreases, while $f_{e P} / f_{s}$ tends to $f_{P} / f_{s}$ and the associated peak value increases, proving the distance-dependent behavior. For $k=0$ there is no coupling between the readout coil and the sensor coil, and in that case, the height of the peak close to $f / f_{S}=1$ vanishes and only the peak at $f_{p} / f_{S}$ can be measured. Figure $2 \mathrm{~b}$ shows the effect of different values of $C_{P} / C_{S}$ at fixed $k=0.2$. From the results, it can be noticed that for increasing $C_{P} / C_{s}$, the ratio $f_{m P} / f_{s}$ moves towards values lower than 1 , while the ratio $f_{e p} / f_{s}$ tends to 1 .
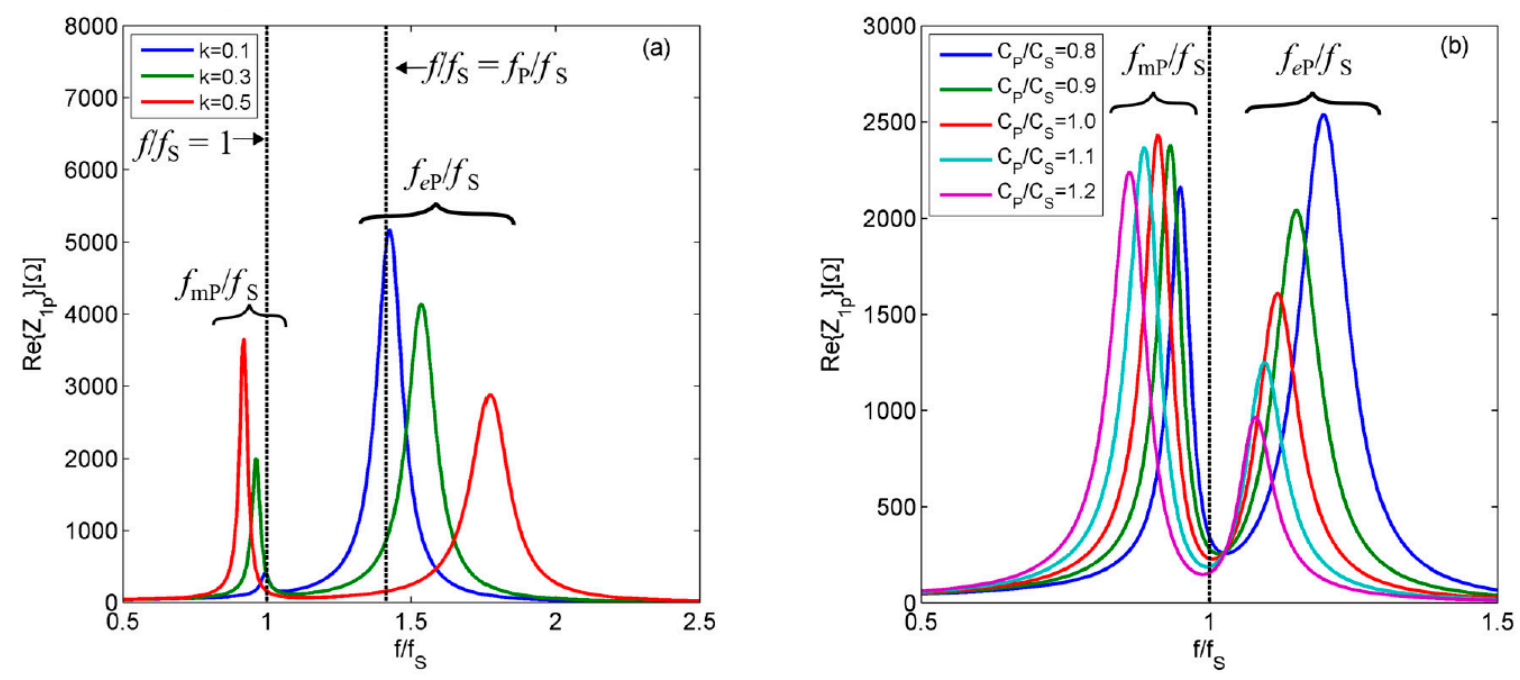

Figure 2. (a) $\operatorname{Re}\left\{Z_{1 P}\right\}$ versus the normalized frequency $f / f_{s}$ for different values of $\mathrm{k}$ and for $C_{P} / C_{S}=0.5$.

(b) $\operatorname{Re}\left\{Z_{1 P}\right\}$ versus the normalized frequency $f / f_{S}$ for different values of $C_{P} / C_{S}$ and for $k=0.2$.

To avoid the dependence of $f_{m P}$ on $C_{P}$ and hence on the distance, the proposed compensation circuit of Figure $1 \mathrm{~b}$ is connected in parallel to the readout coil. The circuit operates as a Negative Impedance Converter (NIC) providing the equivalent impedance $Z_{E q}=V_{1} / I_{E q}=1 /\left(j 2 \pi f\left(-C_{C}\right)\right.$ ), where the negative capacitance $-C_{C}=-C_{A} R_{F} / R_{G}$ can be tuned through $R_{F}$ to compensate and possibly cancel $C_{P}$.

\section{Circuit Prototype and Experimental Results}

The compensation circuit of Figure $1 \mathrm{~b}$ has been built by using a high-bandwidth operational amplifier (AD8045). Figure 3a shows the prototype and the experimental setup adopted to validate the proposed technique. The measured equivalent parameters for the readout and sensor coils are $L_{1}$ $=8.17 \mu \mathrm{H}$ and $R_{1}=3.12 \Omega$, and $L_{2}=8.50 \mu \mathrm{H}$ and $R_{2}=3.20 \Omega$ respectively. For the capacitive sensor, a capacitance $C_{S}=99 \mathrm{pF}$ has been adopted which from (1) gives for the LC resonant circuit $f_{S}=5.48$ $\mathrm{MHz}$ and $Q_{s}=91.5$. 

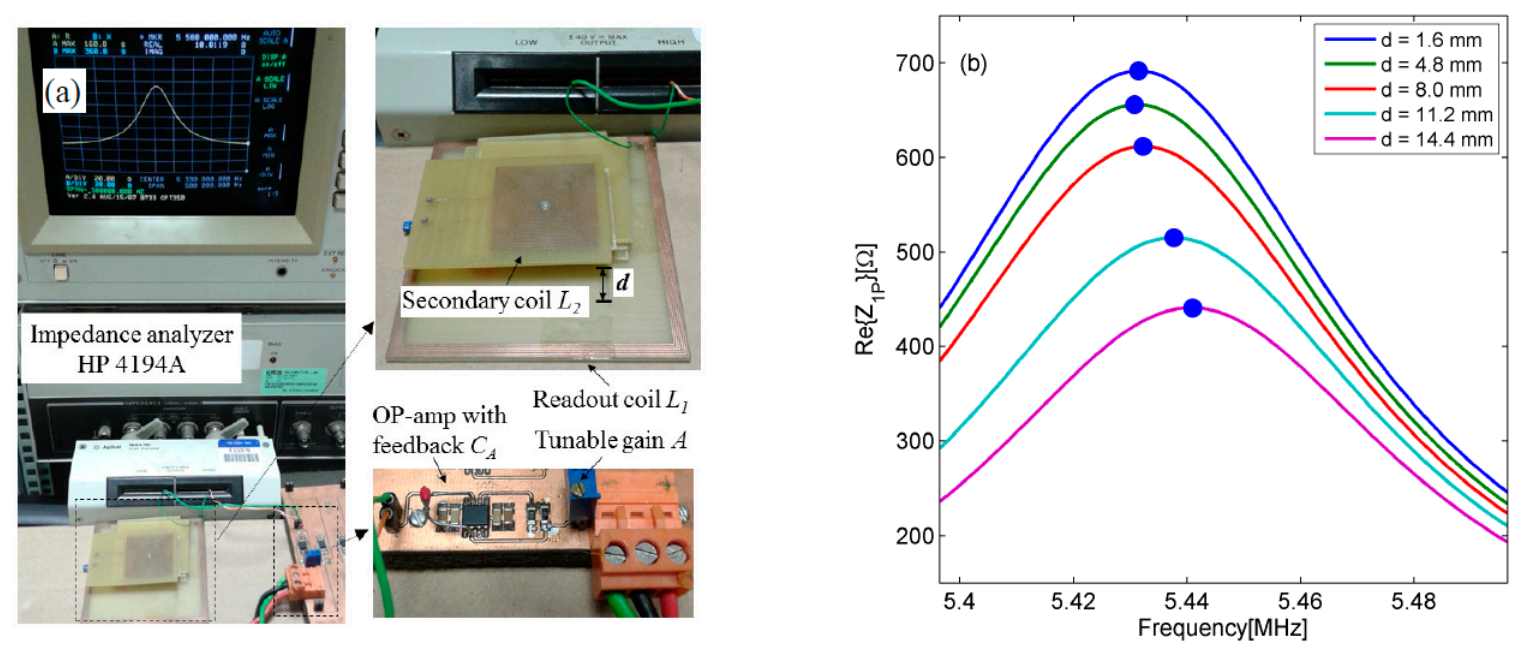

Figure 3. (a) Pictures of the experimental setup. (b) $\operatorname{Re}\left\{Z_{1 P}\right\}$ measured near $f_{S}$ for different values of the interrogation distance $d$ considering a parasitic capacitance $C_{P}=47 \mathrm{pF}$ and without the compensation circuit applied.

To validate the numerical analysis of Section 2 , Figure $3 b$ shows the measured $\operatorname{Re}\left\{Z_{1 P}\right\}$ around $f_{s}$ at five different distances when a total parasitic capacitance of $C_{P}=47 \mathrm{pF}$ is present. It can be observed that, in agreement with the predictions of Figure $2 \mathrm{a}$, the frequency of the maximum of $\operatorname{Re}\left\{Z_{1 P}\right\}$ shifts towards higher frequencies for increasing distances, i.e., for decreasing values of $k$. Figure 4a shows the results of the same test with the compensation circuit tuned for the cancellation of $C_{P}$ activated. Figure $4 \mathrm{~b}$ compares the cases of Figures $3 \mathrm{~b}$ and $4 \mathrm{a}$, showing the relative frequency deviation $\Delta f / f=10^{6}\left(f_{m P}-f_{s}\right) / f_{s}$ as a function of the estimated coupling factor $k$. With the compensation circuit connected, a maximum relative deviation $\Delta f / f$ less than $200 \mathrm{ppm}$ is achieved.
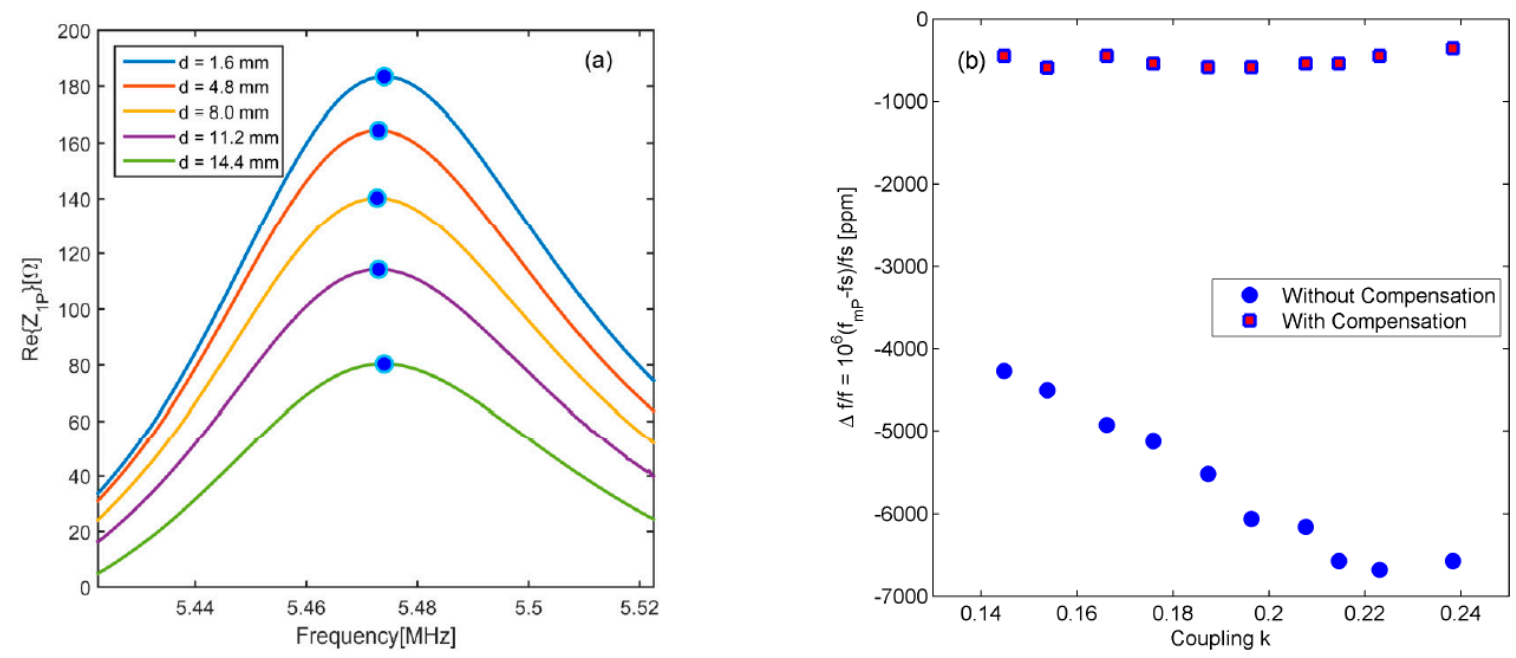

Figure 4. (a) $\operatorname{Re}\left\{Z_{1} p\right\}$ measured near $f_{S}$ for different values of the interrogation distance $d$ considering a parasitic capacitance $C_{P}=47 \mathrm{pF}$ with the compensation circuit applied. (b) Comparison of the relative frequency deviation $\Delta f / f=10^{6}\left(f_{m P}-f_{s}\right) / f_{s}$ with and without the compensation circuit applied.

\section{Conclusions}

The effect of a parasitic capacitance in parallel to the readout coil for contactless measurement of LC passive sensors has been investigated. Numerical analyses and experimental tests show that the parasitic capacitance introduces a dependence of the measured resonant frequency on the interrogation distance. A capacitance compensation circuit is proposed and validated to counteract the effect of the parasitic capacitance, outperforming equivalent systems based on the impedance phase-dip measurement proposed in the literature. 


\section{References}

1. Huang, Q.A.; Dong, L.; Wang, L.F. LC Passive Wireless Sensors Toward a Wireless Sensing Platform: Status, Prospects, and Challenges. J. Microelectromech. Syst. 2016, 25, 822-840, doi:10.1109/JMEMS.2016.2602298.

2. Nopper, R.; Niekrawietz, R.; Reindl, L. Wireless Readout of Passive LC Sensors. IEEE Trans. Instrum. Meas. 2010, 59, 2450-2457, doi:10.1109/TIM.2009.2032966.

3. Demori, M.; Masud, M.; Baù, M.; Ferrari, M.; Ferrari, V. Passive LC sensor label with distance-independent contactless interrogation. In Proceedings of the 16th IEEE SENSORS Conference, Glasgow, UK, 30 October-1 November 2017; doi:10.1109/ICSENS.2017.8234410.

4. Ferrari, M., Baù, M., Tonoli, E., Ferrari, V. Piezoelectric resonant sensors with contactless interrogation for mass-sensitive and acoustic-load detection. Sensors and Actuators, A: Physical 2013, 202, 100-105, doi:10.1016/j.sna.2013.04.029.

5. Demori, M.; Baù, M.; Ferrari, M.; Ferrari, V. Electronic Technique and Circuit Topology for Accurate Distance-Independent Contactless Readout of Passive LC Sensors. Int. J. Electron. Commun. (AEÜ) 2018, 92 , 82-85, doi:10.1016/j.aeue.2018.05.019.

6. Demori, M., Baù, M., Ferrari, M., Ferrari, V. Interrogation techniques and interface circuits for coil-coupled passive sensors. Micromachines 2018, 9, 449, doi:10.3390/mi9090449.

(C) 2018 by the authors. Licensee MDPI, Basel, Switzerland. This article is an open access article distributed under the terms and conditions of the Creative Commons Attribution (CC BY) license (http://creativecommons.org/licenses/by/4.0/). 